\title{
RECOVERED MEMORIES OF ABUSE IN MENTAL ILLNESSES
}

\author{
Solomons T.H.* \\ Department of Psychiatry, Faculty of Medicine, General Sir John Kotelawala Defence University (KDU), Sri \\ Lanka
}

\begin{abstract}
In most mental illnesses, abuse is considered an etiological factor, as a significantly high number of patients report memories of being abused. Yet, there is also a strong evidence base which suggests that recovered memories can be highly unreliable and that they can be creations of the current cognitive biases of individuals. Borderline personality disorder and dissociative disorders have long been linked to a history of abuse. In the current paper, the author discusses three patients; two diagnosed with Borderline personality disorder and the other diagnosed with a dissociative identity disorder. These patients were treated by the author in the private sector and analysis of the weekly treatment records were used for the findings of the current paper. All these patients were females who started treatment in their teenage years. All exhibited a treatment-resistant clinical picture and experienced many short-spaced relapses. After the lapse of about six months into psychotherapy, they accidentally discovered a strong memory of an abuse incident, which could not be traced to any known circumstances of their lives. The memory was highly unlikely to have occurred in reality. Yet, the discovery of the memory and subsequent cognitive processing of the implications and the visual content of these memories marked a notable improvement in the patient. With further treatment, all three were in the remission stage. Therefore, the author feels that patients may have abusive memories, which may or may not be necessarily linked to real life circumstances, yet may strongly influence the patient's symptoms. However, despite the validity of these memories, it is clear that these memories should be treated as significant by clinicians who treat mental illnesses.
\end{abstract}

Keywords: mental illnesses, abusive memories, psychotherapy, recovered memories

\section{Introduction}

The author is a registered clinical psychologist, who provides services both in the private sector and the public sector. Author provides services for individuals with many different mental illnesses. According to current research literature, a high proportion of individuals who come across mental health services have a history of abuse in childhood. A strong link between childhood abuse and maltreatment has been observed in the development of mood disorders. Further, it has been noted that there is poor treatment response in adults with mood disorders who have a history of childhood maltreatment and abuse. (Lippard \& Nemeroff, 2020) (Paz, Jones, \& Byrne, 411-421)

Despite these pieces of evidence, the validity of such memories which are related in retrospect has been often questioned. There is a strong evidence base in the literature of cognitive psychology, which suggests that recovered memories which are related in retrospect can be highly unreliable. Further, 
there is evidence that these memories could also be creations of the current cognitive biases of the individual. Especially, if the memories were recovered later in therapy, and if the patient was not aware of such abuse at the beginning of the therapy, they are believed to be controversial. This is due to the fact that historically, such recovered memories which were recovered during the course of therapy have led to legal action. (Dodier et al., 2019). Sexual abuse has been the most frequently reported type of abuse in memories recovered during therapy. It has further been observed that recovery of such memories was frequent in cognitive behavioural treatment, as much as traumafocused therapies. (Dodier et al. 2019). Yet systematic researches on these are scarce as most research has to depend on anecdotal evidence and case reports as researching such memories have serious ethical implications. Therefore, sharing such case reports are of value for clinicians who are involved in providing treatment to these patient populations.

Researchers point to two types of memories recovered in the course of psychotherapy. Some memories are recovered in the course of suggestive psychotherapy, which is intended on recovering early experiences of abuse. Other types of memories are recovered spontaneously, without any prompting from the therapist. Memories recovered without suggestive psychotherapy in the absence of the prompting of the therapists are believed to be more reliable. (Geraerts, 2011)

The current paper discusses three cases where the patient recovered abuse-related memories, during a psychotherapeutic intervention primarily based on Cognitive Behaviour Therapy (CBT) with integrated techniques of Mindfulness Based Cognitive Therapy (MBCT), which are primarily cognitive approaches. Patients discussed in the current paper did not have prior knowledge of being abused and did not receive any prompting from the therapist to discover such a memory.

\section{Research Methods}

The cases were selected from the clinical records of the author. The author has selected three cases where the patient did not possess any prior knowledge of being abused in childhood. In both cases, the abuse-related memories were recovered spontaneously, without any prompting within the course of psychotherapy. Weekly treatment records were analysed for the purpose of the current paper.

Verbal consent is obtained from each patient when they commence treatment on the possible use of clinical records in teaching exercises and research publications that may be done in future. Further, the patients are educated on their rights of withdrawal of such consent. The cases included in the current study are of patients who did not have any objection to such use of their data. Necessary written permission has also been obtained when required. Further, their identity has been adequately concealed in the current paper, so as to protect their confidentiality

\section{Case Series}

\section{Case A}

"A" was a patient diagnosed with borderline personality disorder. The patient was 20 years at the time she came into treatment with the author. She was from a middle-class socioeconomic background. She had constant conflicts with her parents. Despite the conflicts, she was close to her mother but had 
resentments with the father due to the experience of the father abusing her mother during her childhood. She has 2 suicidal attempts when she started therapy with the author.

She had a memory of brutally being stabbed and killed in an isolated house. From what could be gathered from the memory, the house was a tiny hut, in the middle of a jungle, with many trees surrounding it. The patient saw herself as older/ mature than she was at the time. She was capable of describing the imagery in detail. She had strong feelings of fear, loneliness and a general feeling of pain associated with this memory. These were feelings that constantly surfaced in high intensity during her suicidal attempts.

The possible cognitive bias that could have affected this memory was her memory of physical abuse of her mother by the father.

\section{Case B}

"B" was a patient diagnosed with dissociative identity disorder. "B" was 15 at the time she started treatment with the author. She was from an upper socioeconomic class background. She was close to her parents and her brother. She had no known history of abuse when she started treatment. She had a memory of being beaten up or physically attacked by $5-6$ men with covered faces. It was unclear if the attackers were masked or not. The patient was able to describe the image in detail. She also had a strong feeling of fear, loneliness and a general feeling of pain associated with the image. These were prominent when she had relapses. There were no cognitive biases that could be found which affected the memory.

\section{Case C}

"C" was an 18-year-old female, from an upper-class socioeconomic background referred for treatment by a psychiatrist for the treatment of depression. Upon further assessment, it was clear that the patient also qualified for a diagnosis of borderline personality disorder (BPD). The patient was adopted immediately after birth, of which the patient did not know. The patient had a conflictual relationship with her mother and missed her father who had passed away a few years back. She discovered a memory of her adult self (which appeared to be much older than herself at the time) being stabbed by a man whose face was clear to her. Despite the image being clear, she could not recognize the person and she could not recall having met the man within her lifetime. The author could not find any cognitive biases that could have affected the memory.

\section{Case comparison}

\section{Similarities}

Case A, B and C were females who started treatment with a mental health professional in their teenage years. All three were on pharmacological treatment by a consultant psychiatrist and had been to more than one therapist for psychotherapy.

There was a treatment-resistant clinical picture with many short-spaced relapses. All three discovered an abuse-related memory close to six months into therapy. The discovery was sudden and the memory was described in vivid detail with strong visual content. Their memories could not be linked to any 
known circumstances of their lives. What was most striking was, all described themselves as older or mature in memory than what they were at the time.

After the visual content of the imagery was processed and their related cognitive content was explored, there was a marked improvement in all the patients. All entered a remission stage and they were followed up for one year with booster sessions. During follow up period, they were able to maintain their gains in therapy and their level of functioning had improved.

\section{Discussion and implications for clinicians}

The fact that the patients' entering the remission stage being closely linked in time to the discovery of the memory may suggest that the particular memory had a strong impact on the symptoms experienced by the patients. Further, this may suggest that such memories may have important implications on the etiology, precipitation and perpetuating of mental illnesses, yet such inferences are difficult to make without systematic research into these.

Further, as all the patients had been to more than one therapist before coming into contact with the author, this may suggest a difficulty in building rapport with a therapist. Difficulty in building relationships in adults who have experienced abuse in childhood has been well documented in the research literature. Thus, it could be that irrespective of the reliability of the memory, abuse-related memories may pose difficulties in forming adult relationships.

Further, the fact that these memories cannot be termed as childhood memories, as all three saw themselves as older than they were at the time may have important implications. Patient B termed it as a past life memory, placing it in her Buddhist religious context. This helped the patient come to terms with the fact that she could not connect it to any known circumstances of her current life. Thus recognizing that there may be memories that may not be connected to a patient's known life history and helping them assimilate these memories to their socio-cultural belief system may also be an important part of treatment.

There may be abuse related memories that are unreliable and may not be understood in relation to the real-life circumstances of the patients. Yet these may have a strong impact on the symptoms of mental illnesses and their course of illness. Therefore, it is of utmost importance for clinicians to treat such memories as significant in the treatment process. As research indicates such memories are influenced by the current cognitive biases of the individuals, it could also be that these memories are cognitive constructions of the current emotional symptoms of the patients. However, what should be emphasized is that these memories should be treated as significant during the treatment of mental illnesses. This approach can be different if the memories are explored for legal action or to determine criminal responsibility.

In addition to this, as all three patients presented with a treatment-resistant clinical picture, it may imply that it would always be better for the clinicians to explore the presence of visual cognitive content which may be related to abuse when they encounter a treatment-resistant clinical picture. 
In both cases, the feelings that were associated with the image were also the feelings that were associated with relapse and suicidal attempts. This may also mean that, when there are severe emotional symptoms, the cognitive create visual content to match those symptoms.

\section{Conclusions and Further research}

Based on the current paper, recovered Memories of abuse that psychiatric patients have, appear to affect their psychological functioning, irrespective of whether they occurred in their life or not. Therefore, it is important that clinicians treat such memories as significant for the treatment provided. Addressing the implications of such memories during psychotherapy can significantly affect the treatment outcomes.

As the current paper is based on limited data of three cases, it is difficult to make any definitive conclusions. Therefore, systematic research on the presence of such memories in psychiatric populations would be extremely valuable. As psychiatric populations can be vulnerable, designing a research methodology to investigate these without breaching ethical standards would be of paramount importance. Especially, as some psychiatric patients can be highly suggestive, direct inquiry of abusive memories may lead them onto believing that they have been abused, which may affect the patient's condition. One important thing for clinicians do to would be to maintain a registry of cases where psychiatric patients spontaneously recover abuse-related memories during therapy and analyze the cumulative data.

\section{References}

Dodier, O., Patihis, L., \& Payoux, M. (2019). Reports of recovered memories of childhood abuse in therapy in France. Memory, 27(9), 1283-1298.

Geraerts, E. (2012). Cognitive underpinnings of recovered memories of childhood abuse. True and False Recovered Memories, 175-191.

Lippard, E. T., \& Nemeroff, C. B. (2020). The devastating clinical consequences of child abuse and neglect: increased disease vulnerability and poor treatment response in mood disorders. American journal of psychiatry, 177(1), 20-36.

Paz, I., Jones, D. and Byrne, G., 2005. Child maltreatment, child protection and mental health. Current Opinion in Psychiatry, 18(4), pp.411-421. 